ISSN 0103-5150

Fisioter. Mov., Curitiba, v. 26, n. 2, p. 259-269, abr./jun. 2013

Licenciado sob uma Licença Creative Commons

\title{
Respostas fisiológicas em adolescentes obesos submetidos à broncoprovocação com solução salina hipertônica
}

\author{
Physiological responses in obese adolescents submitted \\ to bronchial provocation with hypertonic saline
}

\author{
Fabrício Cieslak $^{[\mathrm{a}]}$, Wendell Arthur Lopes ${ }^{[\mathrm{b}]}$, Antonio José Grande ${ }^{[\mathrm{c}]}$, Paulo Cesar Kussek ${ }^{[\mathrm{d}]}$, \\ Nelson Augusto Rosário Filho ${ }^{[\mathrm{e}]}$, Neiva Leite ${ }^{[\mathrm{f}]}$
}

[a] Doutorando em Atividade Física e Saúde pela Universidade Federal do Paraná (UFPR), membro do Núcleo de Pesquisa em Qualidade de Vida (NQV-UFPR), Curitiba, PR - Brasil, e-mail: facieslak@gmail.com

[b] Doutorando em Biodinâmica do Movimento Humano pela Universidade Estadual de Campinas (Unicamp), membro do Núcleo de Pesquisa em Qualidade de Vida (NQV-UFPR), Curitiba, PR - Brasil, e-mail: wendell@ufpr.br

[c] Mestre em Educação Física pela Universidade Metodista de Piracicaba (Unimep), Piracicaba, SP - Brasil, e-mail: grandeto@gmail.com

[d] Mestre em Saúde da Criança e do Adolescente pela Universidade Federal do Paraná (UFPR), responsável pela Unidade de Pneumologia Pediátrica do Hospital Pequeno Príncipe, Curitiba, PR - Brasil, e-mail: pneumologia@hpp.org.br

[e] Doutor em Saúde da Criança e do Adolescente pela Universidade Estadual de Campinas (Unicamp), professor titular da Universidade Federal do Paraná (UFPR), Curitiba, PR - Brasil, e-mail: nelson.rosario@ufpr.br

[f] Doutora em Saúde da Criança e do Adolescente pela Universidade Federal do Paraná (UFPR), líder do Núcleo de Pesquisa em Qualidade de Vida (NQV-UFPR), Curitiba, PR - Brasil, e-mail: neivaleite@gmail.com

\section{Resumo}

Introdução: A hiper-responsividade brônquica é a resposta do aumento dos mecanismos fisiológicos protetores das vias aéreas em indivíduos atópicos e não atópicos. Além disso, a magnitude da hiperresponsividade aérea apresenta influência conforme o grau deobesidade. Objetivo: Avaliar asrespostas fisiológicas em adolescentes obesos submetidos a hiper-responsividade brônquica a solução salina hipertônica. Materiais e métodos: Estudo descritivo, transversal e correlacional composto por 15 adolescentes obesos, de ambos os gêneros. O diagnóstico de asma foi realizado por meio de histórico clínico e questionário ISAAC, e a obesidade pelo IMC acima do percentil 95. Utilizou-se o teste de broncoprovocação por solução salina hipertônica para avaliação da hiper-responsividade brônquica, considerando positiva uma diminuição do volume expiratório forçado no primeiro segundo $\left(\mathrm{VEF}_{1}\right)>15 \%$ do valor pré-solução salina e a intensidade da hiper-responsividade 
brônquica foi calculada pela queda percentual máxima do $\mathrm{VEF}_{1}\left(\% \mathrm{Queda}_{\text {máx }} \mathrm{VEF}_{1}\right)$. Foram utilizados o teste $\mathrm{t}$ independente ou U de Mann-Whitney e a correlação de Spearman rho $(\mathrm{p}<0,05)$. Resultados: Não foram encontradas diferenças significativas entre os asmáticos e não asmáticos para as variáveis antropométricas, espirométricas, lipídicas e hemodinâmicas. Verificaram-se moderadas correlações positivas e diferenças significativas entre o \% Queda $a_{\text {max }} \mathrm{VEF}_{1}$ com o IMC $(\mathrm{p}=0,040)$ e IMC escore-Z $(\mathrm{p}=0,028)$. Foram detectadas correlações negativas e diferenças significativas para a $\mathrm{Queda}_{\text {máx }} \mathrm{VEF}_{1}$ e leucócitos $(\mathrm{p}=0,005)$ e para o \% Queda ${ }_{\text {máx }} \mathrm{VEF}_{1}$ com o sulfato de dehidropiandrosterona $(\mathrm{p}=0,032)$. Conclusão: Pode-se concluir que os adolescentes obesos submetidos a hiper-responsividade brônquica apresentam alterações espirométricas que estão associadas às inflamações sistêmicas da obesidade.

Palavras-chave: Adolescentes. Testes de provocação brônquica. Obesidade.

\section{Abstract}

Introduction: The bronchial hyperresponsiveness is the response of increased physiological protective mechanisms in the airways of atopic and non-atopic individuals. Furthermore, the magnitude of hyperresponsiveness influences provider submits with the degree of obesity. Objective: To evaluate the physiological responses in obese adolescents undergoing bronchial hyperresponsiveness to hypertonic saline. Materials and methods: Cross-sectional and correlation study consisted of 15 obese adolescents of both genders. The diagnosis of asthma was made by clinical history and ISAAC, and obesity as BMI above 95th percentile. We used the bronchial provocation test by hypertonic saline for the assessment of bronchial hyperresponsiveness, considering positive a decrease in forced expiratory volume in one second $\left(F E V_{1}\right)>15 \%$ of pre-saline and the intensity of bronchial hyperresponsiveness was calculated as the percentage fall maximum of FEV $\left(\% F_{1}\right.$ (Fall $\left.{ }_{m a x} F E V_{1}\right)$. We used the independent $T$ or MannWhitney $U$ test and Spearman's rho correlation $(p<0.05)$. Results: There were no significant differences between asthmatics and non-asthmatics for anthropometric, spirometric, lipid and hemodynamic variables. There were positive correlations and significant differences between the \% Fall ${ }_{\max } F E V_{1}$ with BMI $(p=0.040)$ and BMI Z-score $(p=0.028)$. There were detected negative correlations with significant differences for Fall máx $F E V_{1}$ and leukocytes $(p=0.005)$ and the $\%$ Fall $_{\text {máx }} F E V_{1}$ with dehydropiandrosterone sulfate ( $p=0.032)$. Conclusion: It can be concluded that obese adolescents have bronchial hyperresponsiveness presented spirometric changes that are associated with systemic inflammation of obesity.

Keywords: Adolescents. Bronchial provocation tests. Obesity.

\section{Introdução}

A prevalência de excesso de peso tem aumentando substancialmente em diversos países, inclusive no Brasil, sendo considerada uma epidemia de âmbito mundial $(1,2)$. Na população pediátrica, a presença da obesidade está relacionada ao desenvolvimento de doenças cardiovasculares e sintomas de asma $(3,4)$.

A obesidade pode tornar-se um fator de risco para a asma porque o excesso de tecido adiposo no organismo pode ocasionar alterações na mecânica respiratória e na resposta inflamatória das vias aéreas $(3,5,6)$. Nesse sentido, os sintomas de asma são resultantes do aumento da resposta de estruturas fisiológicas de proteção das vias aéreas, denominada "hiper-responsividade brônquica" (7).

Evidências recentes têm demonstrado mecanismos de associação da obesidade e asma com a hiper-responsividade brônquica (8). Nos obesos, as propriedades mecânicas do sistema respiratório são alteradas com o efeito da gordura sobre o diafragma e o tórax, ocasionando uma restrição torácica. Com o bloqueio da excursão do diafragma e a redução da complacência torácica, verificam-se possíveis aumentos da hiper-responsividade aérea (6).

A hiper-responsividade brônquica está relacionada com a morbidade respiratória autorreportada e o diagnóstico clínico de asma (8). Dados epidemiológicos têm demonstrado que a hiper-responsividade 
aérea é aumentada em indivíduos asmáticos e obesos $(9,10,11,12)$. Além disso, a literatura relata que os estudos conduzidos nesse âmbito demonstram uma maior prevalência de asma em indivíduos obesos em comparação com asmáticos não obesos $(4,12)$.

Diversos métodos têm sido utilizados para quantificar a hiper-responsividade, sendo o método de espirometria o mais comumente aceito e associado com a provocação brônquica para induzir a broncoconstrição (10). Os testes de provocação brônquica apresentam duas categorias, os testes "diretos" que incluem os agentes farmacológicos histamina e metacolina, e os testes "indiretos" que envolvem a broncoprovocação por exercício físico, hiperapneia de ar seco, manitol e solução salina hipertônica (9).

Com dificuldade de obtenção dos métodos diretos, os testes de broncoprovocação por exercício físico e solução salina hipertônica têm sido utilizados para avaliar a hiper-responsividade com maior frequência $(4,10,11,12)$. Apesar de serem métodos análogos, o teste de exercício demonstra uma perda hídrica das vias aéreas para o meio exterior decorrente da hiperapneia fisiológica. A inalação de solução salina hipertônica reproduz este mecanismo, com as vantagens de ser realizada com volume corrente e não necessitar de colaboração do paciente em atingir a carga máxima de esforço $(9,10)$.

Embora a prova de broncoprovocação por solução salina hipertônica tenha sido aplicada em investigações para avaliar a hiper-responsividade, as evidências desse efeito na relação entre obesidade e sintomas respiratórios na população pediátrica são limitadas (10-12). Nesse sentido, o presente estudo visou avaliar as respostas fisiológicas em adolescentes obesos submetidos a brocoprovocação por inalação de solução salina hipertônica.

\section{Materiais e métodos}

\section{Planejamento da pesquisa e participantes}

O presente estudo apresentou caráter descritivo, transversal e correlacional e e foi realizado com a participação de 15 adolescentes obesos, de ambos os gêneros, os quais foram classificados de acordo com o índice de massa corporal (IMC), conforme a proposta do Center for Disease Control and Prevention (CDC) (13). O número de sujeitos foi calculado pelo programa GPower 2.0, com base em evidências prévias
(12) para um nível de significância de 0,05 , poder estatístico de 0,8 e magnitude de efeito moderado $(d=0,05)$. Foi estabelecido um número mínimo de dez participantes, recrutados de forma não aleatória e por conveniência, conforme os critérios de classificação de obesidade (13).

Todos os sujeitos receberam individualmente esclarecimentos a respeito dos objetivos, procedimentos utilizados, possíveis benefícios e riscos atrelados à execução do presente estudo, e posteriormente condicionaram a sua participação de modo voluntário, mediante apresentação do Termo de Consentimento Livre e Esclarecido assinado pelos pais ou responsáveis. 0 protocolo de pesquisa do presente estudo foi fundamentado em conformidade com as diretrizes propostas na Resolução n. 196/96, do Conselho Nacional de Saúde, sobre pesquisas envolvendo seres humanos. 0 presente estudo foi aprovado no Comitê de Ética do Hospital de Clínicas da Universidade Federal do Paraná (Protocolo número: 1818.235/2008-11).

Os seguintes critérios de inclusão foram estabelecidos: (a) condição de participação em todas as avaliações; (b) apresentação do termo de consentimento assinado pelos pais ou responsáveis; (c) indivíduos classificados como obesos; (d) autorrelato de nenhuma infecção respiratória nas quatro semanas anteriores ao teste, baseado em exames médicos realizados anteriormente ao início das avaliações; (e) autorrelato de nenhum tratamento medicamentoso e histórico de distúrbios cardiovascular, respiratório, músculo-esquelético e/ou metabólico; (e) autorrelato de não uso de alimentos ou medicamentos com cafeína nas duas horas precedentes à execução dos testes. Foram excluídos os indivíduos que: (a) fossem classificados como pré-púberes na avaliação de maturação sexual.

\section{Avaliações antropométricas e clínicas}

Para mensuração da massa corporal (MC), utilizou-se uma balança digital Plenna, com resolução de $0,1 \mathrm{~kg}$, e determinou-se que o avaliado deveria estar descalço e com o mínimo de roupa possível. A estatura foi aferida utilizando-se um estadiômetro, com resolução de 0,1 cm; para efetuar a medida, o avaliado deveria estar descalço, em apneia inspiratória e com a cabeça posicionada no plano de Frankfort (14). Por intermédio das medidas de MC e estatura, 
calculou-se o IMC. Os critérios adotados para classificação do IMC foram realizados de acordo com os valores críticos para classificação do estado nutricional de crianças e adolescentes propostos pelo CDC (13).

A circunferência abdominal (CA) foi verificada conforme os procedimentos descritos por Callaway et al. (15), estando o avaliado em pé com o abdômen relaxado, os braços descontraídos ao lado do corpo, a fita colocada horizontalmente no ponto médio entre a borda inferior da última costela e a crista ilíaca. Para as mensurações, utilizou-se uma fita antropométrica inelástica, com resolução de $0,1 \mathrm{~cm}$. Os valores da CA foram classificados de acordo com os pontos de corte sugeridos por Fernandez et al. (16).

A avaliação puberal dos indivíduos foi realizada por autoavaliação mediante gravuras conforme os estágios maturacionais propostos por Tanner (17); as meninas foram avaliadas quanto ao desenvolvimento mamário e à pilificação pubiana, e os meninos foram mensurados quanto ao tamanho testicular e à pilificação pubiana. Esse método apresenta concordância satisfatória com a avaliação médica em obesos (18).

As medidas das pressões arteriais sistólica (PAS) e diastólica (PAD) foram realizadas no indivíduo sentado, após 10 minutos de repouso. A pressão arterial foi aferida no braço direito apoiado em nível cardíaco, utilizando-se esfigmomanômetro de mercúrio, do tipo aneroide, previamente calibrado conforme o INMETRO, com o tamanho do manguito apropriado ao braço do indivíduo. 0 manguito foi inflado rapidamente até $30 \mathrm{mmHg}$ acima do desaparecimento do pulso radial e desinflado a uma velocidade de $2-4 \mathrm{mmHg}$ /segundo. A PAS foi identificada pelo aparecimento dos sons e a PAD pelo seu desaparecimento (fase V de Korotkoff). Foram obtidas duas medidas, com intervalos de 2 minutos entre elas, considerando-se a medida mais baixa para análise dos níveis pressóricos dos pacientes. Consideraram-se como limítrofes ou aumentados os valores acima ou iguais ao $90^{\circ}$ percentil, para idade e sexo. Os valores obtidos foram classificados conforme as tabelas específicas em percentis para crianças e adolescentes (19).

\section{Avaliação da composição corporal}

A composição corporal foi avaliada pelo método da impedância bioelétrica (BIA), com o aparelho apropriado (Biodynamics ${ }^{\circledR}$ tetrapolar). Realizou-se procedimento nos indivíduos em jejum de 10 a 12 horas, no período da manhã, em decúbito dorsal, após o esvaziamento vesical. Os eletrodos foram posicionados na superfície dorsal das mãos e dos pés, respectivamente, próximo às articulações metacarpo-falangeanas e metatarso-falangeanas e medialmente entre as proeminências distais do rádio e da ulna, entre os maléolos tibial e fibular. Foram obtidos os valores de resistência e calculadas a massa livre de gordura (MLG) e a massa gorda por meio das equações validadas por Houtkooper et al. (20).

\section{Exames laboratoriais}

As amostras sanguíneas foram coletadas no período da manhã, após 12 horas de jejum, para a realização de hemograma e dosagens de glicose, insulina, colesterol total (CT), lipoproteínas de alta densidade (HDL-C), lipoproteínas de baixa densidade (LDL-C) e triglicérides (TG). Após 120 minutos da ingestão de solução contendo 1,75 gramas de glicose/kg (até no máximo 75 gramas), foram coletadas amostras sanguíneas para dosagens de glicemia e insulinemia. Os níveis de tiroxina livre (T4 livre) e sulfato de dehidropiandrosterona (S-DHEA) foram mensurados em jejum. As concentrações plasmáticas de $\mathrm{CT}$, TG e de HDL-C foram determinadas em $\mathrm{mg} / \mathrm{dl}$ por meio de teste colorimétrico enzimático (CHODPAP) (Laboratório Merck, Darmstadt, Alemanha; Laboratório Roche, Indianápolis, IN, EUA). O LDL-C foi calculado pela equação de Friedwald et al. (21), em $\mathrm{mg} / \mathrm{dl}$ : LDL-C = CT $-($ HDL-C + TG/5). Foram considerados como referência os valores para lipídios apresentados na I Diretriz de Prevenção da Aterosclerose na Infância e na Adolescência (22).

Os níveis de glicemia foram determinados com o método enzimático (Glicose Oxidase - Labtest) e classificando-se os indivíduos conforme a Associação Médica Brasileira e Conselho Federal de Medicina (23). A insulina foi dosada pela técnica de quimioluminescência por imunoensaio $\mathrm{em}_{\mathrm{u}} \mathrm{u} / \mathrm{ml}$, em equipamento automatizado, utilizando como referência $11,9 \mathrm{u} \mathrm{u} / \mathrm{ml}$. Para o cálculo da resistência à insulina, utilizou-se a Homeostasis Model Assessment (HOMA-IR) descrito por Matthews et al. (24). Para avaliação da sensibilidade à insulina, foi utilizada o Quantitative Insulin Sensivity Check Index (QUICKI) descrito por Katz et al. (25). 
Avaliação da função pulmonar e hiper-responsividade brônquica a solução salina hipertônica

Os participantes foram orientados a: não ingerir café, chá ou refrigerante com cafeína duas horas antes da avaliação; suspender o uso de broncodilatadores de ação curta e longa 12 horas antes e o uso de anti-histamínicos de ação curta e longa, respectivamente, 48 horas e 5 dias antes da avaliação. Os avaliados não poderiam apresentar sintomas de infecção viral de vias aéreas superiores nas últimas quatro semanas e não estar em crise de asma.

A função pulmonar foi mensurada por espirômetro (Microlab - 2000); os participantes deveriam se manter em posição sentada e fazer uso de clipe nasal. As variáveis pulmonares mensuradas foram a Capacidade Vital Forçada (CVF) e o volume expiratório forçado no primeiro segundo $\left(\mathrm{VEF}_{1}\right)$, em litros. Realizaram-se três manobras espirométricas; foi selecionada aquela com os maiores valores do $\mathrm{VEF}_{1}$ e CVF. Calcularam-se os percentuais dos valores preditos do $\mathrm{VEF}_{1}$ e CVF para a idade e sexo, conforme Polgar; Promodhat (26) e a relação $\mathrm{VEF}_{1} / \mathrm{CVF}$. Antes dos testes, foram explicadas as técnicas de manobras necessárias à realização do teste de função pulmonar, com pelo menos uma espirometria antes da basal, para ter resultados reprodutivos, seguindo as diretrizes para teste de função pulmonar (7) e não apresentando $\mathrm{VEF}_{1}$ menor que $75 \%$ no dia do teste.

Iniciou-se o teste pela inalação de solução salina com nebulizador ultrassônico em débito máximo, com volume inicial de $240 \mathrm{ml}$ e peso inicial de 760 gramas do conjunto, nebulímetro, solução hipertônica e mangueira. 0 nebulímetro foi pesado antes e após cada nebulização, com reposição de solução hipertônica ao volume e peso inicial; isso porque o nível de fluido dentro do recipiente interfere no débito. 0 participante, sentado confortavelmente, era encorajado a manter boa postura, para proporcionar a adequada administração da solução salina. Solicitou-se respirar normalmente pelo bucal com válvula expiratória, com clipe nasal, evitando hiperventilação. A instrução era para deglutir o excesso de saliva evitando, assim, que a saliva entrasse no tubo. Elevação do tubo acima da altura da boca do paciente e inversão bucal com a válvula expiratória para baixo também foram procedimentos necessários a evitar que a saliva entrasse no tubo inalatório. A solução que permanecesse no tubo inalatório ao final da inalação era reincluída no volume do nebulímetro para posterior pesagem. A solução salina era retirada do frasco com seringa de 25 $\mathrm{ml}$ usando agulha estéril, mantendo em ar ambiente, mesmo durante a execução do teste.

Cada indivíduo realizou a inalação por $0,5,1,2$, 4 e 8 minutos, totalizando 15,5 minutos, em técnica de uso de inalações crescentes, encerrando-se o teste, quando havia declínio do $\mathrm{VEF}_{1}$ igual ou maior a $15 \%(11,12)$, ou com o tempo máximo acumulado 15,5 minutos. 0 total da substância inalada foi verificado pesando-se o nebulímetro com a mangueira após cada nebulização, verificando-se a diferença em relação ao peso inicial. Além das mensurações dos volumes expiratórios forçados no primeiro segundo $\left(\mathrm{VEF}_{1}\right)$ nos testes de hiper-responsividade, após a inalação salina, calculou-se a queda percentual máxima do $\mathrm{VEF}_{1}\left(\%\right.$ Queda $_{\text {máx }} \mathrm{VEF}_{1}$ ), utilizando o cálculo da diminuição percentual do $V_{E} F_{1}$ pós-salina em relação ao valor pré-salina pela seguinte equação: \% Queda $_{\text {máx }} \mathrm{VEF}_{1}=\left(\left(\mathrm{VEF}_{\text {1pós-salina mais baixo }}-\mathrm{VEF}_{1 \text { pré-salina }}\right) \mathrm{x}\right.$ $100) / \mathrm{VEF}_{1 \text { pré-salina. }}$

\section{Tratamento estatístico}

Para tratamento dos dados, foi empregada a estatística descritiva com a utilização de frequências, percentuais, mediana, medidas de tendência central e variabilidade (média e desvio padrão) para a caracterização dos participantes do estudo. Para a verificação da normalidade do conjunto de dados, foi utilizado o teste de Shapiro-Wilk. Para verificar as possíveis diferenças entre os grupos, foi aplicado o teste t independente (dados paramétricos), e quando necessário, o teste U de Mann-Whitney (dados não paramétricos). Na análise da associação do $\operatorname{VEF}_{1}(\mathrm{l}) \mathrm{e}$ \% Queda máx $\mathrm{VEF}_{1}$ com as variáveis antropométricas, lipídicas e hemodinâmicas, foi utilizada a correlação de Spearman rho. Os dados foram analisados no software estatístico Statistical Package for the Social Sciences (SPSS, versão 13.0) for Windows, com um nível de significância estipulado em $\mathrm{p}<0,05$ para todas as análises.

\section{Resultados}

Foram estudados 15 adolescentes púberes (nove meninas e seis meninos), cujas características antropométricas e pressão arterial das avaliações iniciais 
são apresentadas na Tabela 1, conforme a presença ou não de asma. Não foram encontradas diferenças significativas entre as médias dos asmáticos e não asmáticos. As médias das variáveis antropométricas estão acima dos valores recomendados para adolescentes eutróficos. Os valores das pressões arteriais demonstraram que $20 \%$ dos indivíduos apresentavam medidas hipertensivas, porém as médias não diferiram significativamente entre os grupos.

Na Tabela 2 apresentados valores iniciais dos exames laboratoriais nos adolescentes obesos estudados. Não foram encontradas diferenças significativas entre os grupos, porém os valores de glicemia, insulinemia, CT, HDL-C, LDL-C e triglicérides - em 20\%, 73\%, 13\%, $80 \%, 7 \%$ e $47 \%$ dos sujeitos, respectivamente - encontram-se acima dos valores recomendáveis para adolescentes eutróficos.
São apresentados na Tabela 3 os valores iniciais das variáveis espirométricas dos indivíduos avaliados. Não foram encontradas diferenças significativas das variáveis espirométricas entre os grupos.

Na Tabela 4 apresentam-se os valores das relações entre as variáveis espirométricas e as variáveis antropométricas. Foram verificadas moderadas correlações positivas e com diferenças significativas entre o \% Queda máx $V F_{1}$ com o IMC ( $\left.p=0,040\right)$ e IMC escore-Z $(p=0,028)$.

Na Tabela 5 são apresentados os valores das relações entre as variáveis espirométricas e as variáveis laboratoriais. Foram verificadas moderadas correlações negativas e com diferenças significativas para a Queda ${ }_{\text {máx }} V_{E F}$ e leucócitos $(\mathrm{p}=0,005)$ e para o \% Queda $_{\text {máx }} V_{E F}$ e S-DHEA $(\mathrm{p}=0,032)$.

Tabela 1 - Variáveis antropométricas e pressões arteriais dos indivíduos

\begin{tabular}{lccc}
\hline Variáveis & Asmático $(\mathbf{n}=\mathbf{6})$ & Não asmático $(\mathbf{n}=\mathbf{9})$ & Total $(\mathbf{n}=\mathbf{1 5})$ \\
\hline Idade $($ meses) & $\Delta$ & $166,88 \pm 21,43$ & $160,26 \pm 24,37$ \\
Idade $(\mathrm{anos})^{\Delta}$ & $150,33 \pm 27,04$ & $13,90 \pm 1,78$ & $13,35 \pm 2,03$ \\
MC $(\mathrm{kg})$ & $12,52 \pm 2,25$ & $80,72 \pm 11,95$ & $81,11 \pm 13,84$ \\
Estatura (cm) & $81,70 \pm 17,53$ & $163,34 \pm 7,57$ & $161,91 \pm 8,41$ \\
IMC $\left(\mathrm{kg} / \mathrm{m}^{2}\right)$ & $159,76 \pm 9,92$ & $30,19 \pm 3,45$ & $30,87 \pm 4,23$ \\
IMC escore-Z & $31,89 \pm 5,38$ & $3,16 \pm 1,12$ & $3,55 \pm 1,43$ \\
$\mathrm{CA}(\mathrm{cm})^{\Delta}$ & $4,13 \pm 1,75$ & $98,58 \pm 8,59$ & $100,50 \pm 10,96$ \\
\% de Gordura & $103,36 \pm 14,21$ & $36,57 \pm 5,11$ & $37,32 \pm 4,93$ \\
MG (kg) & $38,46 \pm 4,85$ & $29,46 \pm 6,95$ & $30,38 \pm 7,82$ \\
MLG (kg) & $31,77 \pm 9,51$ & $50,72 \pm 7,29$ & $50,52 \pm 8,21$ \\
PAS (mmHg) & $50,22 \pm 10,18$ & $100,22 \pm 13,09$ & $104,26 \pm 14,79$ \\
PAD (mmHg) & $110,33 \pm 16,26$ & $68,66 \pm 10,39$ & $70,13 \pm 11,79$ \\
\hline
\end{tabular}

Legenda: ${ }^{\Delta}=$ Variáveis que não apresentaram distribuição normal; ${ }^{*}=p<0,05$.

Fonte: Dados da pesquisa.

Tabela 2 - Características dos parâmetros laboratoriais dos indivíduos

(Continua)

\begin{tabular}{lccc}
\hline Variáveis & Asmático $(\mathbf{n}=\mathbf{6})$ & Não asmático $(\mathbf{n}=\mathbf{9})$ & Total $(\mathbf{n}=\mathbf{1 5})$ \\
\hline CT $(\mathrm{mg} / \mathrm{dL})$ & $147,16 \pm 12,38$ & $152,44 \pm 29,55$ & $150,33 \pm 23,68$ \\
HDL-C $(\mathrm{mg} / \mathrm{dL})$ & $41,50 \pm 6,80$ & $42,66 \pm 8,01$ & $42,20 \pm 7,32$ \\
LDL-C $(\mathrm{mg} / \mathrm{dL})$ & $81,33 \pm 7,00$ & $85,77 \pm 21,96$ & $84,00 \pm 17,27$ \\
Triglicérides $(\mathrm{mg} / \mathrm{dL})$ & $122,33 \pm 47,54$ & $119,88 \pm 61,13$ & $120,86 \pm 54,26$ \\
Glicemia $(\mathrm{mg} / \mathrm{dL})$ & $94,50 \pm 6,77$ & $95,44 \pm 7,38$ & $95,06 \pm 6,91$
\end{tabular}


Tabela 2 - Características dos parâmetros laboratoriais dos indivíduos

(Conclusão)

\begin{tabular}{lccc}
\hline Variáveis & Asmático $(\mathbf{n}=\mathbf{6})$ & Não asmático $(\mathbf{n}=\mathbf{9})$ & Total $(\mathbf{n}=\mathbf{1 5})$ \\
\hline Glicemia 120 $(\mathrm{mg} / \mathrm{dL})$ & $95,66 \pm 21,07$ & $99,88 \pm 10,78$ & $98,20 \pm 15,15$ \\
Insulina $(\mu \mathrm{Ul} / \mathrm{mL})$ & $23,30 \pm 14,94$ & $18,81 \pm 7,43$ & $20,60 \pm 10,79$ \\
Insulina 120 $(\mu \mathrm{Ul} / \mathrm{mL})$ & $55,88 \pm 43,09$ & $42,88 \pm 27,16$ & $48,08 \pm 33,59$ \\
HOMA-IR & $5,57 \pm 3,81$ & $4,40 \pm 1,66$ & $4,87 \pm 2,67$ \\
QUICKI & $0,31 \pm 0,03$ & $0,31 \pm 0,02$ & $0,31 \pm 0,02$ \\
Eosinófilos & & $1,66 \pm 1,32$ & $3,00 \pm 3,44$ \\
Leucócitos & $5,00 \pm 4,73$ & $7382,22 \pm 1913,52$ & $8297,33 \pm 2500,15$ \\
TSH & $9670,00 \pm 2806,28$ & $2,39 \pm 1,43$ & $2,26 \pm 1,26$ \\
S-DHEA & $2,07 \pm 1,05$ & $112,07 \pm 64,30$ & $98,35 \pm 60,19$ \\
T4 Livre & $76,40 \pm 51,53$ & $1,25 \pm 0,23$ & $1,27 \pm 0,19$ \\
\hline
\end{tabular}

Legenda: ${ }^{\Delta}=$ Variáveis que não apresentaram distribuição normal, ${ }^{*}=p<0,05$.

Fonte: Dados da pesquisa.

Tabela 3 - Variáveis espirométricas basais e após teste de broncoprovocação por solução salina

\begin{tabular}{|c|c|c|c|}
\hline Variáveis & Asmático $(n=6)$ & Não asmático $(\mathrm{n}=9)$ & Total $(n=15)$ \\
\hline VEF $_{1}$ basal (I) & $2,80 \pm 0,74$ & $3,11 \pm 0,51$ & $2,99 \pm 0,60$ \\
\hline $\operatorname{VEF}_{1}$ basal (\% pred) & $90,57 \pm 9,96$ & $95,24 \pm 14,06$ & $91,00 \pm 12,41$ \\
\hline CVF basal (I) & $3,42 \pm 1,04$ & $3,63 \pm 0,60$ & $3,55 \pm 0,78$ \\
\hline CVF basal (\% pred) & $103,48 \pm 13,93$ & $104,13 \pm 13,27$ & $103,88 \pm 13,04$ \\
\hline $\mathrm{VEF}_{1} / \mathrm{CVF}$ basal & $82,96 \pm 7,19$ & $86,14 \pm 7,67$ & $84,62 \pm 7,40$ \\
\hline $\mathrm{VEF}_{1}$ Queda $_{\text {máx }}(\mathrm{l})$ & $2,65 \pm 1,13$ & $3,24 \pm 0,45$ & $3,00 \pm 0,82$ \\
\hline$\%$ Queda $_{\max } \mathrm{VEF}_{1}{ }^{\Delta}$ & 2,41 & $-2,99$ & $-2,99$ \\
\hline
\end{tabular}

Legenda: ${ }^{*}=p<0,05 ;^{\Delta}=$ Variáveis que não apresentaram distribuição normal.

Fonte: Dados da pesquisa.

Tabela 4 - Correlações entre as variáveis espirométricas após teste de broncoprovocação por solução salina e as características antropométricas e pressões arteriais

\begin{tabular}{|c|c|c|}
\hline Variáveis & $\operatorname{VEF}_{1}$ Queda $_{\text {máx }}(\mathrm{I})$ & \% Queda ${ }_{\text {máx }} V_{E F}$ \\
\hline MC (kg) & 0,102 & 0,364 \\
\hline Estatura (cm) & 0,412 & 0,016 \\
\hline IMC $\left(\mathrm{kg} / \mathrm{m}^{2}\right)$ & $-0,320$ & $0,536^{*}$ \\
\hline IMC escore-Z & $-0,478$ & $0,564^{*}$ \\
\hline $\mathrm{CA}(\mathrm{cm})$ & $-0,356$ & 0,500 \\
\hline$\%$ de Gordura & $-0,494$ & 0,396 \\
\hline MG (kg) & $-0,309$ & 0,471 \\
\hline MLG (kg) & 0,261 & 0,193 \\
\hline PAS (mmHg) & $-0,065$ & 0,190 \\
\hline PAD (mmHg) & $-0,158$ & 0,368 \\
\hline
\end{tabular}

Legenda: ${ }^{*}=p<0,05$.

Fonte: Dados da pesquisa. 
Tabela 5 - Correlações entre as variáveis espirométricas após teste de broncoprovocação por solução salina e os parâmetros laboratoriais

\begin{tabular}{lcc}
\hline Variáveis & VEF $_{1}$ Queda $_{\text {max }}(\mathrm{I})$ & \% Queda $_{\text {max }} V_{\text {EF }}{ }_{1}$ \\
\hline CT $(\mathrm{mg} / \mathrm{dL})$ & $-0,054$ & 0,171 \\
HDL-C $(\mathrm{mg} / \mathrm{dL})$ & $-0,353$ & 0,339 \\
LDL-C $(\mathrm{mg} / \mathrm{dL})$ & 0,139 & $-0,154$ \\
Triglicérides $(\mathrm{mg} / \mathrm{dL})$ & 0,005 & 0,164 \\
Glicemia $(\mathrm{mg} / \mathrm{dL})$ & 0,071 & 0,176 \\
Glicemia $120(\mathrm{mg} / \mathrm{dL})$ & $-0,092$ & 0,181 \\
Insulina $(\mu \mathrm{Ul} / \mathrm{mL})$ & $-0,461$ & 0,334 \\
Insulina 120 $(\mu \mathrm{Ul} / \mathrm{mL})$ & $-0,303$ & $-0,009$ \\
HOMA-IR & $-0,349$ & $-0,350$ \\
QUICKI & 0,349 & 0,350 \\
Eosinófilos & $-0,338$ & $-0,120$ \\
Leucócitos & $-0,685^{\star}$ & 0,264 \\
TSH & $-0,347$ & 0,332 \\
S-DHEA & 0,389 & $-0,594^{*}$ \\
T4 Livre & $-0,287$ & $-0,279$ \\
\hline
\end{tabular}

Legenda: ${ }^{*}=p<0,05$.

Fonte: Dados da pesquisa.

\section{Discussão}

Evidências da literatura relatam que a obesidade precede o desenvolvimento da asma; além disso, a prevalência de asma é identificada com maior frequência em indivíduos obesos comparado aos não obesos $(1,4,6)$. Os mecanismos propostos para a influência da obesidade sobre o desenvolvimento da asma incluem a inflamação das vias aéreas, fatores mecânicos, aumento da hiper-responsividade aérea, fatores hormonais, doenças de refluxo gastroesofágico, distúrbios do sono e etiologias comuns entre as doenças $(1,8)$.

A prova de broncoprovocação por solução salina é muito utilizada em investigações epidemiológicas para avaliar a prevalência de asma $(10,11)$. 0 desenvolvimento de testes de broncoprovocação utilizando aerosóis de solução salina hipertônica foi apresentado como consequência de investigações para análise da hiperpneia de ar seco causada pelo estreitamento das vias aéreas por aumento da osmolaridade das vias aéreas superiores (9). A solução salina hipertônica tem sido apresentada como uma ferramenta para identificar a hiper-responsividade brônquica e para efetivar a combinação com a indução de cuspe para mensuração do número de células inflamatórias realizada concomitantemente à hiper-responsividade aérea (27). A solução salina foi desenvolvida inicialmente com a utilização de uma solução de 3,6\% e, subsequentemente, com $4,5 \%$ de solução salina (27). A vantagem da broncoprovocação salina está no estreitamento aéreo durante o teste de modo mais do que o provocado por exercício ou hiperpneia voluntária, nos quais a broncoconstrição ocorre depois que o teste é encerrado $(27,28)$.

Na comparação das variáveis antropométricas, espirométricas, lipídicas e hemodinâmicas em adolescentes obesos submetidos a broncoprovocação por solução salina hipertônica não foram detectadas diferenças significativas entre os grupos. Os avaliados deste estudo apresentaram valores percentuais adequados em relação aos do predito para estatura e sexo e relação $V_{E F} / C V F$ acima de $80 \%$. Isso ocorreu tanto nos indivíduos asmáticos como nos não asmáticos e não foram encontradas diferenças significativas, fato que demonstra a normalidade da função pulmonar conforme os critérios da American Thoracic Society (7).

Evidências têm relatado que o aumento de gordura corporal pode causar um decréscimo da 
função ventilatória $(1,12)$. No estudo de Ulger et al. (12) o decréscimo de $V_{E F}$ e CVF demonstrou uma forte associação com o excesso de gordura subcutânea obtido pelo método de dobras cutâneas comparado à relação cintura x quadril. Em nossa pesquisa, as variáveis espirométricas demonstraram associação com as variáveis antropométricas IMC e IMC escore-Z. Esses resultados sugerem que as alterações extrínsecas e intrínsecas sobre o pulmão podem reduzir a capacidade de difusão e ocasionar um decréscimo na área alveolar relativa ao volume pulmonar (12).

Na avaliação dos indivíduos quanto aos parâmetros laboratoriais, foram encontradas moderadas correlações negativas para $\mathrm{VEF}_{1} \mathrm{Queda}_{\text {máx }}$ com os leucócitos. Esses dados demonstram que a inflamação sistêmica incluindo elevações nos leucócitos sanguíneos aumenta similarmente em pacientes com asma e sem asma em resposta ao exercício (29). Durante o exercício ocorre um aumento da perda de água das vias aéreas para o meio exterior decorrente da hiperpneia fisiológica. 0 estímulo osmolar parece não agir diretamente em receptores do músculo liso, mas provavelmente estimula a liberação de mediadores das células da mucosa brônquica que direta ou indiretamente causam a contração do músculo liso (27). Apesar de não efetuarmos a broncoprovocação por exercício em nossa pesquisa, devemos considerar que o método de solução salina hipertônica apresenta um princípio de aplicabilidade similar e de maior segurança em relação ao exercício $(27,28)$.

Foram verificadas relações das variáveis espirométricas com o sulfato de dehidropiandrosterona nos sujeitos deste estudo. A literatura indica que em sujeitos asmáticos, o sulfato de dehidropiandrosterona é um forte indicador de supressão adrenocortical no tratamento com inalação de esteroides (30). Além disso, ressalta-se que a função pulmonar pode ser influenciada por fatores lipídicos e hemodinâmicos, uma vez que, a relação entre asma e obesidade pode ser explicada por fatores na inflamação sistêmica relacionadas à obesidade incluindo mudanças nos hormônios reguladores (1).

A interpretação de nosso estudo deve ser efetuada com cautela diante de algumas implicações clínicas. 0 delineamento amostral de seleção não aleatória dos sujeitos com características étnicas próprias pode ter resultado em um viés de seleção em nossa pesquisa; além disso, o baixo número de participantes pode ter prejudicado a apresentação de alguns resultados referentes às correlações. $\mathrm{Na}$ pesquisa de Ulger et al. (12), os valores espirométricos basais foram menores em obesos quando comparados ao grupo controle. Tal fator pode ser entendido como outra limitação de nosso estudo, de modo que a ausência de grupo controle pode dificultar a generalização dos resultados.

As contribuições clínicas do presente estudo demonstram que a mensuração da hiper-responsividade brônquica em adolescentes obesos por intermédio método de inalação salina hipertônica pode apresentar eficácia adequada para avaliação das respostas fisiológicas. Não obstante, esse método de broncoprovocação apresenta baixo custo, requer poucos equipamentos e necessita de mínima cooperação dos avaliados durante sua execução $(10,12)$.

\section{Considerações finais}

De modo geral, os adolescentes obesos asmáticos e não asmáticos não apresentaram diferenças para as variáveis antropométricas, espirométricas, lipídicas e hemodinâmicas. Verificaram-se relações do IMC e IMC escore-Z com as variáveis espirométricas. Além disso, as relações das variáveis espirométricas com os leucócitos e com o sulfato de dehidropiandrosterona demonstram que a hiper-responsividade brônquica está associada à inflamação sistêmica na obesidade. Desse modo, para confirmar esses resultados são necessárias novas investigações, realizando-se análises de outros parâmetros fisiológicos referentes às respostas de hiper-responsividade brônquica em adolescentes obesos, que controlem as limitações apontadas nesta análise e determinem indicativos para a utilização de testes de função pulmonar para indivíduos com obesidade.

\section{Referências}

1. Shore SA. Obesity and asthma: possible mechanisms. J Allergy Clin Immunol. 2008;121(5):1087-93. doi:10.1016/j.jaci.2008.03.004.

2. Instituto Brasileiro de Geografia e Estatística - IBGE. Pesquisa de orçamentos familiares [acesso 8 set. 2006]. Disponível em: http://www.ibge.gov.br 
3. Leite N, Milano GE, Cieslak F, Lopes WA, Rodacki A, Radominski RB. Effects of physical exercise and nutritional guidance on metabolic syndrome in obese adolescents. Rev Bras Fisioter. 2009;13(1):73-81. doi:10.1590/S1413-35552009005000009.

4. Lopes WA, Radominski RB, Rosário NA Filho, Leite N. Exercise-induced bronchospasm in obese adolescents. Allergol Immunopathol. 2009;37(4):175-9. doi: 10.1016/j.aller.2009.03.001.

5. Li AM, Lex C, Zacharasiewicz A, Wong E, Erin E, Hansel $\mathrm{T}$, et al. Cough frequency in children with asthma: correlation with lung function, exhaled nitric oxide, and sputum eosinophil count. Thorax. 2003;58(11):974-8. doi:10.1136/thorax.58.11.974.

6. Poulain M, Doucet M, Major GC, Drapeau V, Series F, Boulet LP, et al. The effect of obesity on chronic respiratory diseases: pathophysiology and therapeutic strategies. Can Med Assoc J. 2006; 174(9): 1293-9. doi:10.1503/cmaj.051299.

7. Sociedade Brasileira de Pneumologia e Tisiologia - SBPT. Diretrizes para teste de função pulmonar. J Pneumol. 2002;28(supl. 3):1-238.

8. Shore SA, Fredberg J. Obesity, smooth muscle, and airway hyperrresponsiveness. J Allergy Clin Immunol. 2005;115(5):925-7. doi:10.1016/j.jaci.2005.01.064.

9. Leuppi JD, Brannan JD, Anderson SD. Bronchial provocation tests: the rationale for using inhaled mannitol as a test for airway hiperresponsiveness. Swiss Med Wkly. 2002;132(13-14):151-8.

10. Kussek P, Rosario NA Filho, Cat M. Bronchial hyperresposiveness to hypertonic saline challenge in children and adolescents. J Bras Pneumol. 2006;32(3):195201. doi:10.1590/S1806-37132006000300004.

11. Riedler J, Gamper A, Eder W, Oberfeld G. Prevalence of bronchial hiperresposiveness to $4,5 \%$ saline and its relation to asthma and allergy symptons in Austrian children. Eur Respir J. 1998;11(2):355-60. doi:10.11 83/09031936.98.11020355.

12. Ulger Z, Demir E, Tanaç R, Goksen D, Gulen F, Darcan S, et al. The effect of childhood obesity on respiratory function testes and airway hiperresponsiveness. Turk J Pediat. 2006;48(1):43-50. PMid:16562785.

13. Kuczmarski RJ, Ogden CL, Grummer-Strawn LM, Flegal KM, Guo SS, Wei R, et al. CDC growth charts: United States. Adv Data. 2000;314:1-27. PMid:11183293.
14. Gordon CC, Chumlea WC, Roche AF. Stature, recumbent length and weight. In: Lohman TG, Roche AF, Martorell R. Anthropometric standardization reference manual. Champaign: Human Kinetics Books, 1988.

15. Callaway CW, Chumlea WC, Bouchard C, Hilmes JH, Lohman TG, Martin AD, et al. Circumferences. In: Lohman TG, Roche AF, Martorell R. Anthropometric standardization reference manual. Champaign: Human Kinetics, 1988.

16. Fernandez JR, Redden DT, Pietrobelli A, Allison DB. Waist circumference percentiles in nationally representative samples of african-american, european-american, and mexican-american children and adolescents. J Pediat. 2004;145(4):439-44. doi:10.1016/j. jpeds.2004.06.044.

17. Tanner JM. Normal growth and techniques of growth assessment. Clin End Metab. 1986;15(2):411-51. doi:10.1016/S0300-595X(86)80005-6.

18. Leite N, Milano GE, Lazarotto L, Lopes MFA, Pilotto V, Bento PCB, et al. Avaliação e auto-avaliação puberal em crianças e adolescentes obesos. Rev Motriz. 2009;5(3):641-7.

19. Sociedade Brasileira de Cardiologia - SBC. V diretrizes brasileiras de hipertensão arterial. Rev Bras Hipert. 2006;13(4):256-312.

20. Houtkooper LP, Going SB, Lohman TG, Roche AF, Van Loan M. Bioelectrical impedance estimation of fat-free body mass in children and youth: a cross validation study. J Appl Physiol. 1992;72(1):366-73.

21. Friedwald WT, Levy RI, Fredrickson D. Estimation of the concentration of low-density lipoprotein cholesterol in plasma, without use of the preparative ultracentrifugue. Clin Chem. 1972;18(6):499-502.

22. Sociedade Brasileira de Cardiologia - SBC. I Diretriz de prevenção de aterosclerose na infância e adolescência. Arq Bras Cardiol. 2005;85(6):1-36.

23. Sociedade Brasileira de Diabetes - SBD. Tratamento e acompanhamento do diabetes mellitus. 2006. [Acesso 10 jun. 2008]. Disponível em: http://www. diabetes.org.br

24. Matthews DR, Hosker JP, Rudenski AS, Naylor BA, Treacher DF, Turner RC. Homeostasis model assessment: insulin resistance and beta cell-function from fasting plasma glucose and insulin concentrations in man. Diabetol. 1985;28(7):412-9. doi:10.1007/BF00280883. 
25. Katz A, Nambi SS, Mather K, Baron AD, Follmann DA, Sullivan G, et al. Quantitative insulin sensitivity check index: a simple, accurate method for assessing insulin sensitivity in humans. J Clin Endocr Metab. 2000;85(7):2402-10. doi:10.1210/jc.85.7.2402.

26. Polgar C, Promodhat V. Pulmonary function testing in children: techniques and standards. Philadelphia: WB Saunders Co, 1971. p. 87-212.

27. Anderson SD. Indirect challenge tests: airway hyperresponsiveness in asthma: its measurement an clinical significance. Chest 2010;138(2):25-30. doi:10.1378/ chest.10-0116.

28. Smith CM, Anderson SD. Inhalation provocation tests using non-isotonic aerosols. J Allergy Clin Immunol. 1989;84(5 pt 1):781-90. doi:10.1016/ 0091-6749(89)90309-6.
29. Schwindt CD, Zaldivar F, Wilson L, Leu S-Y, WangRodriguez J, Mills PJ, et al. Do circulating leucocytes and lymphocyte subtypes increase in response to brief exercise in children with and without asthma? Br JSports Med. 2007;41(1):34-40. doi:10.1136/ bjsm.2006.030205.

30. Kannisto S, Korppi M, Remes K, Voutilainen R. Serum dehydropiandrosterone sulfate concentration as an indicator of adrenocortical suppression in asthmatic children treated with inhaled steroids. J Clin Endocrinol Metab. 2001;86(10):4908-12. doi:10.1210/jc.86.10.4908.

Recebido: 10/05/2012

Received: 05/10/2012

Aprovado: 18/03/2013

Approved: 03/18/2013 